\title{
The Effect of Exposure to Social Annotation on Online Informed Consent Beliefs and Behavior
}

\author{
Martina Balestra \\ New York University \\ New York, U.S.A. \\ mb5758@nyu.edu
}

\author{
Orit Shaer \\ Wellesley College \\ Wellesley, U.S.A. \\ oshaer@wellesley.edu
}

\author{
Johanna Okerlund \\ Wellesley College \\ Wellesley, U.S.A. \\ jokerlun@wellesley.edu
}

\author{
Madeleine Ball \\ PersonalGenomes.org \\ Boston, U.S.A. \\ madeleine@personalgenomes.org
}

\author{
Oded Nov \\ New York University \\ New York, U.S.A. \\ onov@nyu.edu
}

\begin{abstract}
In this study we explore the impact of exposure to social annotation, embedded in online consent forms, on individuals' beliefs and decisions in the context of informed consent. In this controlled between-subjects experiment, participants were presented with an online consent form for a personal genomics study. Individuals were randomly assigned to either a social annotation condition that exposed them to previous users' comments on-screen, or to a traditional consent form without social input. We compared participants' perceptions about their consent decision, their trust in the organization seeking the consent, and their actual consent across conditions. While no significant difference was observed between actual consent rates, we found that on average individuals exposed to social annotation felt that their decision was more informed, and furthermore, that the effect of the exposure to social annotation was stronger among users characterized by relatively lower levels of prior privacy preserving behaviors.
\end{abstract}

\section{Author Keywords}

Informed consent; social annotations; social influence; personal genomics

\section{ACM Classification Keywords}

H.5.m. Information interfaces and presentation (e.g., HCI): Miscellaneous;

\section{INTRODUCTION}

Social media, mobile and wearable technology, and connected devices have significantly expanded the

\footnotetext{
Permission to make digital or hard copies of all or part of this work for personal or classroom use is granted without fee provided that copies are not made or distributed for profit or commercial advantage and that copies bear this notice and the full citation on the first page. Copyrights for components of this work owned by others than the author(s) must be honored. Abstracting with credit is permitted. To copy otherwise, or republish, to post on servers or to redistribute to lists, requires prior specific permission and/or a fee. Request permissions from Permissions@acm.org.

$C S C W^{\prime} 16$, February 27-March 02, 2016, San Francisco, CA, USA

Copyright is held by the owner/author(s). Publication rights licensed to ACM.

ACM 978-1-4503-3592-8/16/02...\$15.00

DOI: http://dx.doi.org/10.1145/2818048.2820012
}

opportunities for conducting research online. Already recognized as a rich resource for psychological and social research [31], biomedical research is taking increasing interest in these digital methods. Apple's launch of ResearchKit in April 2015 provides an example of a tool created specifically to facilitate biomedical research through online processes and interactions [3]. The reduced barrier to entry for participation in online biomedical research and the sensitivity of the resultant data highlight the importance of informed consent processes and require us to reevaluate their effectiveness and potential in this new context.

Electronic consent poses new challenges when contrasted to traditional consent processes. Whereas individuals were formerly able to ask questions or engage with a professional in additional face-to-face dialogue, potential online research participants have fewer opportunities to ask questions and express their concerns in real time. Furthermore, the use of certain presentation techniques and design interventions may influence an individual's decision to participate [14; 24], raising concerns regarding voluntariness. In response to these and other concerns, federal agencies are drafting guidelines for electronic consent [21].

While electronic consent can reduce the participantresearcher dialogue, the online environment allows the consent deliberation process to move from solitary to social settings. A computer-supported social environment could enable individuals deliberating on their consent decision to connect with each other, share information, formulate and evaluate different perspectives, and ultimately understand the risks and benefits of the research beyond the scope of one-on-one dialogue with a research staff member.

In this empirical study we build upon prior research on social annotation to examine the effect of exposing participants to a socially augmented online consent form (a "social consent form") on their perceptions and behaviors in the context of informed consent. Existing studies on the relationship between consent form design and potential participants' behaviors have predominantly focused on 
graphical or multimedia interventions to highlight important or complex information and improve comprehension (see [23] for a review). We propose that this approach is insufficient - it assumes that the information in the consent form is exhaustive, and does not necessarily encourage individuals to deliberate and synthesize information in ways that are meaningful to them. Rather, we follow a social perspective on learning which "changes the locus of the learning process from that of the mind of the individual to the participation patterns of individual members of organizations in which learning takes place" [18], much in the same way that research on communications-driven group decision support systems sought to capitalize on the distributed knowledge of many to converge on the best decisions [45].

Building on prior CSCW research on social annotation and social influence, this study is a first step in a research program that aims to develop and evaluate social annotation tools to transform the nature of the informed consent deliberation process from individual to social. To make such evaluation as rigorous as possible, we distinguish between two aspects of social annotation research: (1) evaluating the effect of being exposed to social annotation on user behavior; and (2) evaluating the effect of creating and actively engaging with social annotation on user behavior. These two aspects are inherently related, and finding that exposure to social annotations has an effect on user behavior has implications for developing tools enabling users' creation and engagement with annotations. Taken together, such findings will offer preliminary guidelines for developing tools that add a collaborative dimension to the informed consent deliberation process. Our study addresses the first aspect: we hypothesize that exposure to social annotation in information-dense consent forms will help individuals to capitalize on the knowledge of others to bring to light questions, problems, and concerns they may not have considered on their own. It may also help to recreate aspects of the type of discourse that potential participants would have with research administrators in the face-to-face setting. In doing so, social annotation may ultimately improve an individual's comprehension of the material and the research organization when presented in an online environment, and help them make the right decisions for themselves.

In addition, the findings of the paper contribute to current $\mathrm{CSCW}$ research on social influence by exploring its role in socially enabled, digitally mediated consent processes when explicit organizational and social structures are necessarily missing because of the highly sensitive context of medical research. This study demonstrates how aspects of the consent document, surfaced and discussed online through social annotation, influence users' informed consent deliberation and perceptions.

\section{BACKGROUND}

\section{Application Domain: Personal Genomics}

Traditionally, medical genetic testing targeted individual loci and was performed for specific medical contexts (e.g. when investigating a suspected genetic condition). A medical expert mediated the consent process for testing and returning results. A precipitous decline in the costs of genome-scale testing, however, has led to widespread access of personal genomic data. Several companies currently offer genome-scale testing services directly to consumers. Direct-to-consumer genetic testing (DTCGT) is a relatively new and developing online service, which enables individuals to acquire genetic information without the mandatory involvement of a healthcare provider by sending a saliva sample to a DTCGT company at the cost of a few hundred dollars. DTCGT users are often asked to share their genetic and family history information with biomedical researchers who partner with the DTCGT provider. Genetic results, including traits, ancestry and in some cases, health information, are reported using interactive online applications [47; 48]. With DTCGT, computer-mediated consent and the presentation of results have become core aspects of giving individuals access to their genome-scale test results. At the same time, these aspects raise concerns that policy makers as well as researchers attempt to address [21].

\section{Informed Consent}

The decision to consent to participate in medical research is mediated by two main factors: participants' comprehension of the details of the study and their trust in the research organization [30].

Informed consent consists of four core tenets (disclosure, comprehension, voluntariness, competence) and describes the process of educating individuals on a procedure so that they are able to make a well-reasoned decision about their voluntary agreement to participate $[24 ; 38]$. The moral obligation of consent seekers is widely recognized as providing "those facts that all rational persons would want to know, namely, the various goods and evils that result from alternative modes of treatment, including severity and probability" [12]. Ubel and Lowenstein [53] suggest that this approach falls short of helping individuals make decisions that fit with their values. They propose to find a way to combine medical facts with attributes and considerations that are relevant to participants with suspicions, hopes, fears, and anxieties. With this study we assert that adding a computer-supported social aspect to the consent deliberation process means bringing in other perspectives on what "information" is valuable for informed consent.

\section{Consent Forms}

Prior research on the design of consent forms has not yielded consistent results. Early studies on the design of consent forms focused on text readability [27; 39]. Following the realization that readability does not necessarily relate to comprehension [16], research shifted to 
explore different ways to communicate the content of consent forms and other legal documents. Recent studies on consent form design focus predominantly on the impact of content structure, graphical enhancements, and multimedia on comprehension. Dresden and Levitt [15] demonstrate greater comprehension when a consent form is shortened to contain only details that the researchers believe to be most relevant to a potential participant. In a test comparing comprehension of a traditional consent form and a graphically enhanced form, however, Stiles et al. [51] found no significant difference in the rate of comprehension. Murphy et al. [40] show a significant increase in consent form comprehension scores with a combination of restructured text, simplified vocabulary and sentence structure, and the use of illustrations to communicate key concepts. Dunn et al. [17] found that the participants assigned to read a consent form formatted as a structured, computerized slideshow scored higher in comprehension tests than participants assigned to a traditional consent form condition. Other studies, however, show that replacing a traditional consent form with an interactive computer-based presentation does not result in consistent improvements in comprehension $[2 ; 33]$. Multimedia interventions have used video to replace or complement textual consent forms, though comprehension tests have widely demonstrated that video has little effect on consent form comprehension [2; 25]. To the best of our knowledge, the design for a social consent form introduced in this paper is the first of its kind.

\section{Social Annotation}

Social annotations are constituted of three elements: the resource (i.e. the text in question), the users, and the metadata created by the users. In a paper on the collective dynamics of social annotation, Catutto et al. [8] define social annotation as "freely established associations between web resources and metadata [keywords and descriptive labels, categories, ratings, comments and notes] performed by a community of web users with little or no central coordination" (p. 10511) that captures the relevant collective knowledge of all users. Gao [26] asserts that access to this type of social annotation allows users to discuss content collaboratively and asynchronously, and presents evidence that there is more discussion that is more thoughtful, more focused, and more related to the text when users had access to social annotations. Further, Nelson et al. [41] demonstrate substantial learning effects among participants in exploratory learning tasks who had access to social annotations during a controlled laboratory experiment. Within the context of consent forms, incorporating social information may allow individuals to benefit and learn from others' novel perspectives, knowledge and ideas by encouraging discussion and helping to focus attention on the issues they find important.

Cross and Sproull [11] argue that the value of social information is fundamental and not limited to the online environment. In a qualitative study of information relationships the authors find that individuals tend to seek out relationships that support problem reformulation (in which others help to define or redefine dimensions of a problem not previously considered.) In the context of the social consent form, Cross and Sproull's findings show that individuals would perceive the information relationships embodied in social annotations as valuable resources for vetting the risks and benefits of participation.

Access to socially constructed information can impact the decisions an individual makes in areas ranging from consumer products [28] to travel [55] and security feature adoption [13]. Das et al. [13] find that information exchanges on the topic of security tend to begin with an individual's desire to warn others of immediate or novel threats, or to acquire information useful for understanding a particular system or solving a problem. This suggests to us that participants would be motivated to use social annotations in the context of consent, and that the decisions they make about consenting could be influenced in turn by the knowledge and experiences of others.

When user-contributed information is contributed like this it is not usually policed by a centralized authority [8] and therefore annotations may contain inaccurate information or perceptions. Though Bernstein et al. [6] use the social features of Collabio to show that the tags produced by users had a high-degree of accuracy, they attribute this accuracy to social motivators that prevented serious misuse or offtopic tags. These social motivators may not necessarily exist in a context like medical research where anonymity is not only valued, but also legally mandated. Further, in the absence of personal identifiers, potential participants may perceive certain others as "experts", which are more valuable and more persuasive than others, where they might not necessarily be $[19 ; 32]$.

Any potential for false information can have significant impacts on prospective participants. An individual's ability to respond appropriately to a situation requires the ability to correctly interpret and react to incoming information, particularly in compliance-gaining settings [9]. The individual relying on socially-constructed information may therefore be making decisions based on erroneous information or misplaced beliefs, which can substantially detriment not only the participant, but in cases like genomic research, also participants' ancestors and offspring.

\section{Trust and Social Annotation}

Beyond the effective and appropriate communication of information, previous research shows that trust plays a crucial role in the decision to disclose sensitive information online [35]. Similarly, trusting the physician or research organization plays a fundamental role in the decision to participate in medical research [36]. Following from [37] we take trust in the medical context to be "the expectation that institutions and professionals will act in one's interests" (pg. 661). In this context trust is comprised of five dimensions: expectations about the research organization's competence, the extent to which the organization is 
concerned with their patient's welfare, the organization's control over decision making, the organization's management of confidential information, and the organization's openness in providing and receiving information [37]. In traditional consent seeking procedures the individual examines independently the information provided by the authors of a consent form along these five dimensions before making a decision about consent. By implementing social annotations we enable prospective participants to capitalize on the experiences of others to discern trustworthiness, and therefore add a social perspective to the user's development of trust in the organization seeking consent.

\section{HYPOTHESES}

Deliberating whether to participate in medical research can be an incredibly complex process, though individuals' decision-making abilities are limited [53]. Prior research has shown that in such scenarios, individuals tend to simplify these deliberations by ignoring large amounts of information while focusing on a subset of information relevant to their value system $[52 ; 51]$. In the context of consent, we suggest that social annotations serve to connect individuals' value systems to the content of the consent form in an explicit manner. Prospective participants are able to observe, identify with, and learn from the issues, questions, and concerns raised by previous participants on topics relevant to their values, thus focusing their deliberations around these issues and improving the efficiency and effectiveness of their deliberative process. Ultimately, we believe that this will lead participants to feel more informed about their decision to participate (or not) in medical research. Therefore, our first hypothesis is as follows:

H1a. Participants exposed to social annotations in a consent form will feel more informed in their consent deliberation compared to those not exposed to social annotation.

Furthermore, novices (i.e. individuals new to the subject at hand) tend to have relatively basic mental models compared with experts [50]. Less knowledgeable individuals therefore do not benefit from the predictive and explanatory power of informed mental models for understanding situations and interactions [50] in the way that knowledgeable individuals do. Kittur et al. [29] show that having access to others' mental models and knowledge representations can help individuals to build and refine their own schemas. We therefore hypothesize that these less knowledgeable participants stand to benefit more from the effects of social annotations than knowledgeable participants:

H1b. The effect of exposure to social annotations on feeling informed will be stronger among less knowledgeable participants than the effect on more knowledgeable participants.
Nelson et al.'s [41] show that social annotations can be useful in helping individuals to learn unfamiliar topics. Social annotations provide a mechanism for bringing others' knowledge and insights to bear on difficult-tounderstand topics, allowing participants to capitalize on the collective knowledge of previous participants. Furthermore, the additional information contained in social annotations may function as a "scaffold for learning" [41] (p. 1798). That is, annotations not only contribute previous participants' knowledge of complex, confusing, or obscure information, but can serve as "navigational signposts" [41] (p.1798) that aid subsequent users to navigate these complex concepts. We therefore examine the following hypothesis:

H2. Participants exposed to social annotations will feel that they understand the content of the consent form more than those who were not exposed to social annotations.

Finally, we propose that social annotations can play a role in determining trust a prospective participant may feel towards the organization seeking consent. Prior research explored the role of technology-mediated social influence in protecting users in trust-related situations such as security and privacy threats [13], as well as antisocial or exploitative behavior [22]. Potential concerns shared by prior users about information provided to users, may therefore influence users' perception of the information [34]. Further, prior studies demonstrate that negatively framed information is significantly more effective than positively framed information in shaping users' perceptions [42; 49]. Based on these findings, we hypothesize that negative annotations in the consent form will have a greater (negative) effect on prospective participants' perception of the research organization seeking the consent. We therefore hypothesize the following:

H3. Participants will trust the research organization less when exposed to social annotations in the consent form compared with the control condition.

\section{METHODOLOGY}

\section{Procedure}

We conducted a between-subjects experimental study to explore the effects of exposure to social annotation, embedded in an online consent form for a personal genomics study, on users' beliefs and decisions.

A website was developed specifically for this experiment. A link to the study was made available on Amazon Mechanical Turk and participants were paid $\$ 5.00$ for completing the questionnaires. Participation in the study was limited to English speakers with a record of at least 100 prior tasks at an approval rate exceeding 99\%. Since DTCGT is marketed to the general population, we chose to recruit users via Amazon Mechanical Turk. The population of Amazon Mechanical Turk is diverse and reflective of the general population, which make it a viable venue for data collection $[4 ; 44]$. The choice in high prior approval rate 
and the relatively high pay was made in order to increase the likelihood that participants will be reliable and that they will take their time when considering the various choices they have to make as they go through the study.

Participants were asked to take part in a study seeking to understand how users engage and learn from personal genomic information. Participants were first asked to answer several questions about their Internet usage and complete a tutorial on genomics. They were then asked to review the consent form for an additional study in which they could participate that would result in the mapping of their own genome. Users were randomly assigned to view a control condition (a standard consent form without social annotations) or the intervention condition (a social consent form with annotations containing concerns and questions from the perspective of previous potential study participants.) Figures 1 and 2 demonstrate each condition.

In order to maintain ecological validity, participants were led to believe that the additional genome mapping study was a real study in which they could participate. Participants were told that if they consented, they would be linked to an external page where they would be asked to provide their email address, phone number, and basic health information, and would be contacted by an administrator of the genomics study to coordinate further (figure 4). This deception was used to increase the likelihood that participants would take the time to make an informed and honest decision based on the information provided in the consent form. We did not disclose to participants that the genomic study was fictional until the end of the Mechanical Turk study, when they were told the true objective of the study was to learn about the process of consent. No identifying information (email, phone-number, etc.) was ultimately collected.

\section{Research Instruments}

\section{Privacy Questionnaires}

A privacy questionnaire and personal genomics tutorial preceded the consent form. Hypothesis H1b dealt with the relationship between how knowledgeable a participant was and how informed they felt when they made their decision to consent or not. Because many of the risks and issues with digitally mediated research center on data privacy (particularly in the context of genomics research), we chose to use a measure of pre-existing privacy attitudes and behaviors as the measure of an individual's topical knowledge. We used a standard questionnaire developed by Buchanan et al. [7] based on Westin's privacy index [54]. The survey consists of three short scales measuring privacy related attitudes ('Privacy Concern') and behaviors ('General Caution' and 'Technical Protection').

\section{Genomics Tutorial}

The personal genomics tutorial was comprised of learning materials on the human genome and personal genomics developed by the Personal Genetics Education Project [46]. Participants' understanding of the material was assessed using a short six-question quiz. Participants were then presented with a sample personal genomics report for an imaginary individual named Jamie, followed by another comprehension task. This task was used to demonstrate the type of information provided by genetic testing. Jamie's report was developed for this study using a fictional data set in which sex and ethnicity did not have a specific effect, and was modeled on GET-Evidence [46], Harvard's Personal Genomes Project personal genomics report. Participants were asked to study the report and to answer three comprehension questions. Figure 3 shows the personal genomics report presented to users

\section{Social Consent Form}

Following the genomics tutorial participants were presented with the consent form for an additional optional study in which their genomes would be mapped and their family health history and trait information would be collected online. The study was framed as a voluntary contribution to research (rather than a commercial service in exchange for payment), but those who chose to participate would receive their results in a free, online report. The content of the consent form was based on Office for Human Research Protections guidelines [43] and the 23andMe informed consent document (publicly available online at [1]). Modifications to improve the clarity of the text were made based on feedback provided in pilot tests with other Amazon Mechanical Turk users.

Participants were randomly assigned to either a control consent form condition or an intervention condition. The control condition consisted of the consent form text with minimal graphical interventions (Figure 1). The intervention condition social consent form consisted of the same text in the same format as the control condition, but included callouts containing social annotations in the margins of the screen (Figure 2).

Participants were told that these annotations had been contributed by previous prospective Mechanical Turk participants who had seen the same consent form. In reality, the social annotations were derived from feedback provided by participants during pilot tests and manipulated by the researchers. The previous participants' feedback was edited to reflect an equal balance of positive and negative sentiments in an effort to prevent artificially encouraging participants to consent or not. The annotations included questions, concerns, personal perspectives, and contextual information related to the consent form. Though they were manipulated, deriving the annotations from real content allowed us to use annotations that touched on topics that were likely to be meaningful to current participants. Each comment also included an indicator showing how many other hypothetical study participants "liked" the comments, though participants could neither add comments nor "like" existing comments in this condition. The numbers of "likes" were determined by the researchers to balance positive and negative sentiment. 


\section{What are the risks of participating?}

There are some potential risks to participating in the HCIPGP research study. Please read each of the risks carefully before deciding whether or not to participate.

\section{- Ambiguous or Erroneous Data}

The quality of your genomic analysis and the interpretation of your results are subject to human error. An example of such an error in interpretation is the false association of a disease with a specific gene variant. Furthermore, the ability to sequence the human genome is relatively new. The connection between human genes and human traits is not fully understood. Therefore, much of the data currently available regarding the human genome and how it affects us is ambiguous and inconclusive. This may result in misplaced concern or stress about erroneous or incomplete data.

\section{- Anxiety or Stress due to Discovery of Disease Risk}

You may learn that you or a family member is at increased risk of developing a disease or other trait. This may cause anxiety or stress to you or those with whom you share this information.

Figure 1. Example of control condition of consent form

\begin{tabular}{|c|c|c|}
\hline & $\begin{array}{l}\text { 9. What are the risks of participating? } \\
\text { There are some potential risks to participating in the HCIPGP research study. Please } \\
\text { read each of the risks carefully before deciding whether or not to participate. } \\
\text { - Ambiguous or Erroneous Data }\end{array}$ & \\
\hline $\begin{array}{l}\text { This seems like a big } \\
\text { drawback-if I have to be } \\
\text { stressed about the results, } \\
\text { and they might not even be } \\
\text { right?? } \\
\text { but at the same time, } \\
\text { you would be } \\
\text { contributing to making } \\
\text { those tests better... }\end{array}$ & $\begin{array}{l}\text { The quality of your genomic analysis and the interpretation of your results are } \\
\text { subject to human error. An example of such an error in interpretation is the false } \\
\text { association of a disease with a specific gene variant. Furthermore, the ability to } \\
\text { sequence the human genome is relatively new. The connection between human } \\
\text { genes and human traits is not fully understood. Therefore, much of the data } \\
\text { currently available regarding the human genome and how it affects us is ambiguous } \\
\text { and inconclusive. This may result in misplaced concern or stress about erroneous or } \\
\text { incomplete data. } \\
\text { Anxiety or Stress due to Discovery of Disease Risk } \\
\text { You may learn that you or a family member is at increased risk of developing a } \\
\text { disease or other trait. This may cause anxiety or stress to you or those with whom } \\
\text { you share this information. } \\
\text { Carrier Status } \\
\text { You may learn that you carry a gene variant that may be disadvantageous to your } \\
\text { biological children. These gene variants may be transmitted to your biological }\end{array}$ & $\begin{array}{l}\text { The stress really concerns } \\
\text { me, especially if I couldn't } \\
\text { change or do anything about } \\
\text { it. }\end{array}$ \\
\hline
\end{tabular}

Figure 2. Example of social annotations in social consent form

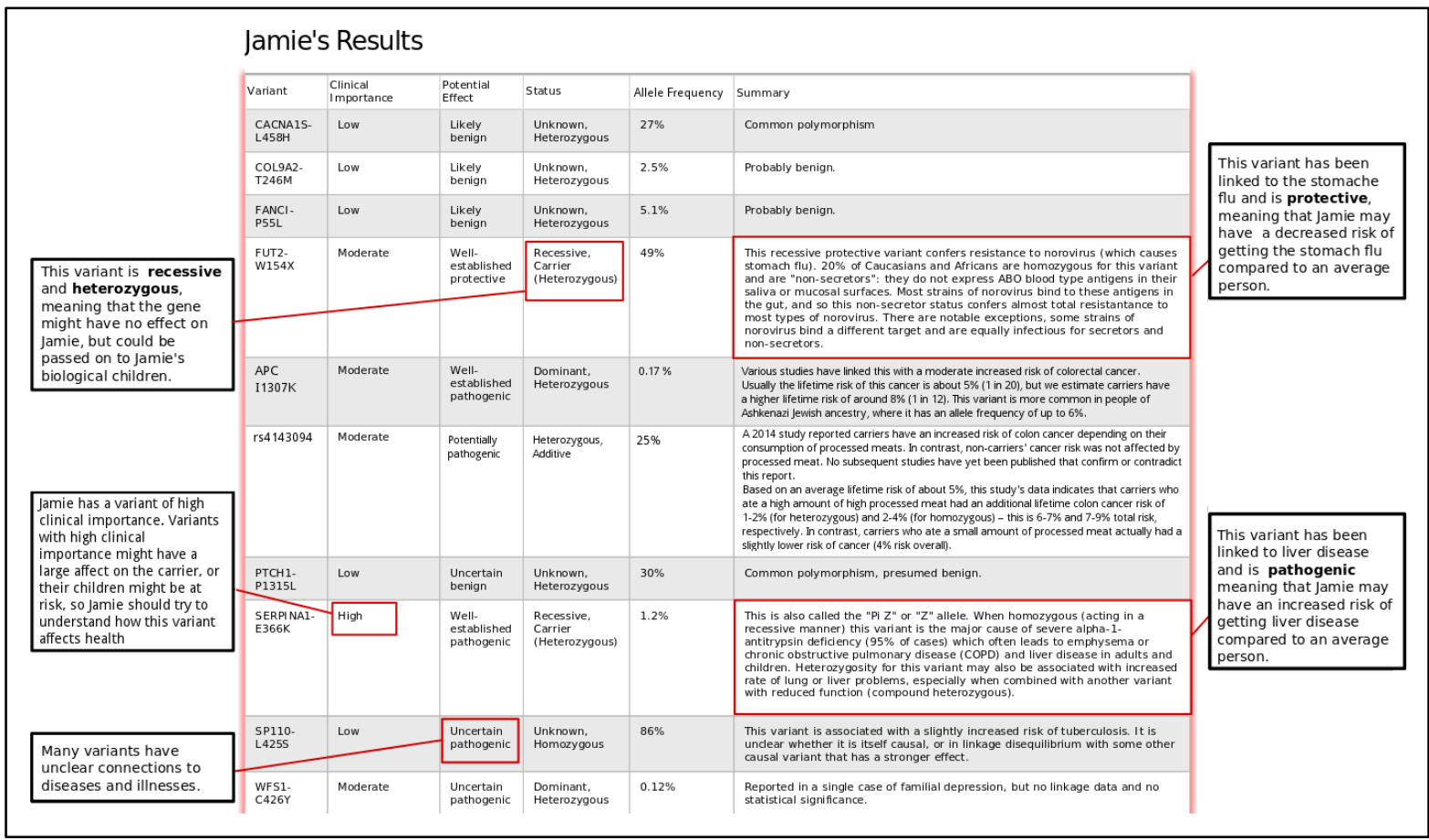

Figure 3. Example of personal genomics report shown to users during training 


\section{CONSENT}

I have read this consent form. All my questions about the study and participation in the study have been answered.

If you choose to consent, in the next pages of this study, you will be linked to an external page and asked to provide your email address, phone number, and basic health information. You will then soon be contacted by an administrator of the study.

I choose to participate in this study and authorize the use and disclosure of my health information to the parties listed in the authorization.

I decline to participate in this study.

Figure 4. Consent question

\section{Measures}

Following their decision about consent to the additional study, users were presented with questions about their deliberative process and perception of the consent form (Table 1). All measures were single-item and self-reported, measured on a 5-point Likert scale (strongly agree strongly disagree). Below we report the questions used to address each hypothesis:

\begin{tabular}{c||l} 
Hypothesis & \multicolumn{1}{c}{ Question } \\
\hline H1a & $\begin{array}{l}\text { "I feel that my decision (to consent or } \\
\text { not) was an informed decision." } \\
\text { "I feel that I understood the material } \\
\text { presented and I have no additional } \\
\text { questions" } \\
\text { "Based on what I have seen and read in } \\
\text { this consent form, I feel like I can trust } \\
\text { the [research organization name, which } \\
\text { is identifying] to use and protect my data } \\
\text { in the ways outlined in the consent } \\
\text { form." }\end{array}$
\end{tabular}

Table 1. Questions used to evaluate each hypothesis

Participants in the social consent form condition were also asked to report on the degree to which they used the social annotations.

\section{Demographics and Disclosure}

Finally, participants were asked demographic questions. They were then informed that the study was fictitious, and that the true research question related to the process of consent and consent forms.

\section{RESULTS}

\section{Demographics}

259 individuals participated in this study, with 138 participants in the control condition and 121 in the social annotation intervention. The average age of participants was 35.5 years old $(\mathrm{SD}=10.7)$, and $58.8 \%$ of participants were female. On average, participants' mean privacy index score was 3.07 (out of 5, $\mathrm{SD}=0.87$ ).

\section{Domain Comprehension}

To ensure sufficient domain understanding we presented participants with training material and comprehension questions. Only 11 (out of 259) answered fewer than 3 out of 6 genome introduction questions, or fewer than 2 out of 3 of the genome report questions, incorrectly. These individuals were removed from the dataset, leaving 248 viable participants (132 in the control condition and 116 in the social intervention). Correlation analysis was used to test whether the domain comprehension scores from the entire population impacted the extent to which they felt their decision was informed (i.e. informed consent). Results of this test were marginally significant $(\mathrm{r}=0.11, \mathrm{p}=0.08)$. Within the subset of viable participants, the correlation analysis showed a significant correlation between comprehension score and the measure of informed decision $(r=0.15, p=0.02)$. The domain comprehension score was therefore controlled for in our analyses going forward.

\section{Time on Consent Form}

Participants spent an average of 326 seconds $(S D=222)$ assessing the consent form (before deciding whether to consent) in the social intervention, and 242 seconds $(\mathrm{SD}=206)$ in the control condition. A t-test comparing the mean times spent in each condition shows that the difference reaches statistical significance $(t(236)=-3.06$, $\mathrm{p}=0.002)$. Additional analysis showed that in the control (i.e. no social annotation) condition, participants who consented $(M=201.13 \mathrm{~s}, \mathrm{SD}=152.63 \mathrm{~s})$ spent significantly more time on the consent form than those who did not consent $(\mathrm{M}=277.93 \mathrm{~s}, \quad \mathrm{SD}=236.54 \mathrm{~s} ; \quad t \quad(121)=2.23$, $\mathrm{p}=0.028)$. The significant difference among those who consented $(\mathrm{M}=338.63 \mathrm{~s}, \mathrm{SD}=211.98 \mathrm{~s})$ and those who did not $(\mathrm{M}=317.30 \mathrm{~s}, \mathrm{SD}=227.71 \mathrm{~s})$ was not found in the social annotation condition $(t(107)=-0.51, \mathrm{p}=0.61)$.

\section{Behavior and Perceptions}

The rate of consent did not differ significantly across conditions $(46.2 \%$ in the social condition vs. $42.2 \%$ in the control condition). However, the social annotation exposure did seem to impact the way participants perceived their decision, supporting hypothesis H1a: on average, participants rated the extent to which they felt their decision (to consent or not) was informed higher in the social annotation condition $(\mathrm{M}=4.46, \mathrm{SD}=0.66)$ than in the control condition $(\mathrm{M}=4.26, \mathrm{SD}=0.95 ; t(234)=-1.85, \mathrm{p}=0.06)$. At the same time, participants rated their perceived understanding of the material marginally higher in the control condition $(\mathrm{M}=4.28, \mathrm{SD}=0.79)$ than in the social condition $(\mathrm{M}=4.07, \mathrm{SD}=0.94, t(225)=1.89, \mathrm{p}=0.06)$, leading us to reject hypothesis H2. Finally, participants rated the extent to which they trust the research organization seeking their consent significantly higher in the control condition $(\mathrm{M}=3.87, \mathrm{SD}=1.01)$ than in the social annotation condition $(\mathrm{M}=3.56, \mathrm{SD}=0.97, t(244)=2.46$, $\mathrm{p}=0.01$ ), in support of hypothesis H3. Table 2 summarizes these results. 


\begin{tabular}{|c|c|c|c|c|c|}
\hline & \multicolumn{2}{|c|}{$\begin{array}{c}\text { Social } \\
\text { annotation }\end{array}$} & \multicolumn{2}{|c|}{ Control } & \multirow[b]{2}{*}{ p value } \\
\hline & M & SD & $\mathbf{M}$ & SD & \\
\hline \multicolumn{6}{|c|}{ perception of decision } \\
\hline $\begin{array}{l}\text { Decision was } \\
\text { informed }\end{array}$ & 4.46 & 0.66 & 4.26 & 0.95 & 0.06 \\
\hline $\begin{array}{l}\text { Made the } \\
\text { right decision }\end{array}$ & 4.56 & 0.51 & 4.56 & 0.64 & $n s$ \\
\hline $\begin{array}{l}\text { Understood } \\
\text { all the } \\
\text { material }\end{array}$ & 4.07 & 0.94 & 4.28 & 0.79 & 0.06 \\
\hline $\begin{array}{l}\text { Trust the } \\
\text { organization } \\
\text { seeking my } \\
\text { consent }\end{array}$ & 3.56 & 0.97 & 3.87 & 1.01 & 0.01 \\
\hline $\begin{array}{l}\text { Read } \\
\text { comments }\end{array}$ & 4.54 & 0.72 & $X$ & $X$ & $X$ \\
\hline $\begin{array}{l}\text { Comments } \\
\text { influenced } \\
\text { decision }\end{array}$ & 2.82 & 1.14 & $X$ & $X$ & $X$ \\
\hline $\begin{array}{l}\text { Comments } \\
\text { helped make } \\
\text { decision }\end{array}$ & 3.15 & 1.13 & $X$ & $X$ & $X$ \\
\hline \multicolumn{6}{|l|}{ Time on task } \\
\hline Time & $326 \mathrm{~s}$ & $222 s$ & $242 \mathrm{~s}$ & $206 \mathrm{~s}$ & 0.002 \\
\hline \multicolumn{6}{|l|}{ Consent rate } \\
\hline Consent & \multicolumn{2}{|c|}{$61(46.2 \%)$} & \multicolumn{2}{|c|}{$49(42.2 \%)$} & $n s$ \\
\hline No consent & \multicolumn{2}{|c|}{71} & \multicolumn{2}{|c|}{67} & \\
\hline
\end{tabular}

Table 2. A comparison between the experimental conditions

To rule out a mere exposure effect (that is, the possibility that just spending more time on a consent form impacts perceptions) independent correlations were constructed comparing the log of the time spent on the consent form to the measures of how informed a participant felt, whether they felt that they understood the material, and the extent to which they trusted the research organization. None of these correlations was statistically significant, indicating that the amount of time a participant spent on the form was unrelated to how they perceived their decision.

Expanding on the analysis of how informed participants felt, an Ordinary Least Squares (OLS) regression model demonstrated that exposure to social annotation had a significant main effect on how informed a participant felt $(\mathrm{B}=0.86, \mathrm{p}=0.03)$, and an individual's privacy index score had a marginally significant effect $(B=0.16, p=0.065)$, as did the interaction of the intervention and the privacy index $(\mathrm{B}=0.23, \mathrm{p}=0.069)$. That is, the intervention had a larger impact on how informed people felt when they engaged in privacy preserving behaviors less frequently, in support of hypothesis H1b. There has been no significant difference between the two conditions in terms of how informed participants felt, among individuals who engaged in privacy preserving behaviors more frequently.

The extent to which participants felt informed in the social condition was also negatively correlated with how influential $(r=-0.20, \mathrm{p}=0.03)$ and how helpful $(r=-0.24$, $\mathrm{p}<0.01)$ they thought the social annotations were. Participants who felt more informed perceived the annotations as less influential and less helpful than people who reported feeling less informed when they made their decision.

\section{DISCUSSION}

Informed Consent and Understanding Consent Material The findings demonstrate that exposure to social annotations results in participants feeling that their decision was more informed, but simultaneously less confident in their understanding of the genomics material presented in the consent form. While this finding may seem contradictory, it contributes to our understanding of the relationship between the comprehension of consent form content and the extent to which people feel that their decision is informed. The discrepancy between how informed a participant felt and how well they understood the material was studied by Ubel and Lowenstein [53], who challenged the assumption that an individual is informed simply because they understand the content of a consent form by rote. Rather, the authors suggested that participants are informed when they comprehend a relevant subset of facts within the context of their particular value system. By this logic - and contrary to conventional wisdom - feeling informed and understanding the material are two separate constructs that do not need to be concordant for either to be true.

Previous research [13] indicates that the most productive role for social annotation in the context of consent is as a decision aid. In particular, people tend to engage around negative aspects of the consent process - specifically, the information provider's desire to warn others about threats and the information seeker's desire to acquire more information about a potential problem. Our findings suggest that drawing attention to limitations in the content of the consent form using social annotation may also highlight limitations in participants' own knowledge. At the same time, sheer awareness of shortcomings in the consent form revealed by the social annotations may contribute to participants simultaneously feeling more informed.

\section{Informed Consent and Privacy Preserving Behaviors}

The findings indicate that the people who benefit most from social annotation in terms of feeling informed were those that reported engaging in privacy preserving behavior the least. This finding could be explained by Cross and Sproull's work [11], which looked specifically at information relationships between experts and nonexperts. They found that information seekers' task-relevant 
expertise had a significant impact on their ability to reformulate and synthesize problems. Participants who were less knowledgeable in the domain of the task were less efficient in their deliberative processes. The authors found that socially constructed information could significantly benefit these non-expert information seeker's deliberative processes, and that the source of the social information did not have to be expert for information seekers to benefit.

We found that while there was no significant difference between users who reported high and low privacy preserving behavior with respect to how informed they felt in the non-social condition, users with low privacy index scores felt significantly more informed than those high privacy scores in the social annotation exposure condition. This finding may be explained using Cross and Sproull's [11] insights. If people who have lower privacy index scores are also less knowledgeable about privacy risks, they stand to gain more from the knowledge and expertise of others, communicated through social annotation. By the same logic, individuals who had high privacy index scores were more knowledgeable in the domain, and benefitted less from additional information contained in the social input.

\section{Trust and Social Annotation}

Participants reported trusting the organization soliciting consent significantly more in the control condition than in the social annotation condition. One explanation for this finding may be that social annotation impacts subjective understanding of consent materials (the extent to which participants felt they understood the content of the consent form); that is, as participants are confronted with the shortcomings in the consent form through social annotation, they become less trusting of the organization. It has been shown that people tend to look to socially constructed information to understand negative aspects of consenting (e.g. risks and consequences) rather than the positive aspects (e.g. the benefits of participation) [13].

\section{Time on Consent Form}

On average, participants spent 84 seconds (34.71\%) more studying the consent form in the social annotation condition than in the control condition. Thus we assume that participants actually read the social annotations in the treatment condition. This is further supported by the fact that social annotation participants reported reading almost all of the social annotations $(\mathrm{M}=4.54, \mathrm{SD}=0.72$, rated between 1 and 5 where 1 is "strongly disagree" and 5 is "strongly agree" with the statement "I read most of the other users' comments"). The difference in time spent on the consent form could also be attributed to increased cognitive engagement in the information-processing task. Previous work shows that targeted information exchange can lead users to become more cognitively engaged in an information processing task, leading to an increase in the proportion of time spent thinking [19].
The findings also show that participants who gave their consent in the control condition spent significantly more time studying the consent form than those who did not consent. This may be because those who consent need more time to deliberate on their decision (compared with those who do not consent). Interestingly, this difference in time deliberating between those who consented and those who did not was not significant in the social intervention. We hypothesize that this discrepancy indicates that participants exposed to social annotations could deliberate on consent more effectively than participants in the control condition. Support for this explanation can be found in [19], where it was suggested that socially constructed information functions as a cognitive aid in the deliberation process, thus helping people to deliberate more effectively.

\section{Research Implications and Contributions}

The findings of this study demonstrate that social annotation techniques implemented in the context of consent forms affect the perception of prospective participants in ways that traditional interface interventions are not likely to achieve. In particular, we showed that turning the informed consent deliberation process from solitary to socially-informed, can result in individuals (and particularly those less knowledgeable on relevant topics) feeling more informed about their decision to consent, and less trusting about the organization seeking the consent.

This study is a first step in developing and evaluating social annotation tools to transform the nature of the informed consent deliberation process, from individual to social. To that end, two complementary aspects of the research into users' perceptions and behavior are needed: evaluating the effect of users' exposure to social annotation; and evaluating the effect of creating and actively engaging with social annotation. These two issues are inherently related, and finding that exposure to social annotations has an effect on user perceptions and behavior has implications for developing effective tools to enable users' creation of, and engagement with, annotations. The study presented here addresses the first aspect and its importance therefore lies not only in demonstrating that exposure to social annotation contributes to shaping users' perception and behavior, but also in laying the foundation for the development of a research-driven collaborative deliberation and decision making tool.

In addition, the findings of the paper contribute to current $\mathrm{CSCW}$ research on social influence. Social influence in online environments and its effect on users in systems such as social recommender systems has been the topic of substantial CSCW research in recent years $[5 ; 14$; 20]. These studies have largely examined the effects of explicit organizational and social structures (that is, relationships, professional hierarchy, physical proximity, etc.) on social influence [10]. Our study contributes to this body of literature by exploring the role of social influence in socially enabled, digitally mediated consent processes 
when these explicit organizational and social structures are necessarily missing because of the highly sensitive context of medical research. Indeed, the findings of this research contribute a new and expanded understanding of the multidimensionality of social annotation in a decision-making context. Social annotation does not merely improve or worsen the user's experience as put forth in existing studies; rather, it changes how participants reflect on their ability to make informed decisions for themselves in complex ways. Where previous studies focused on efficiency gains, learning effects, and accuracy, this study uniquely examines the impact of social annotation on individuals' sentiments and perceptions. We have demonstrated how these complex aspects of the consent document, surfaced and discussed online through social annotation, influence users' informed consent deliberation and perceptions. The system presented here draws on key components of communication-based group decision support systems to deal with asymmetries in the distribution of information that can exist among prospective participants of medical research. This research has provided a proof-ofconcept for a group decision support system in which the focus of deliberation is not among members for the purpose of consensus agreement, but within the individual [45].

\section{Ethical Implications}

Beyond extending our knowledge of the contexts in which social annotation can be used effectively, this study has demonstrates that social annotation interventions can have a real impact in uniquely sensitive and highly regulated settings. In contrast to the spaces where social annotation studies have traditionally been conducted (for example, with respect to consumer products, online search platforms, and security feature adoption) human subjects research requires decisions that are intensely personal and can have substantial ramifications for the individual as well as their offspring. Despite this, the present research demonstrates that strangers' perspectives and knowledge can play a significant role in how individuals make these decisions for themselves, implying a shift in the way that we think about and execute consent-seeking processes.

Researchers have ethical and legal obligations to give individuals an appropriate context for making decisions that may have material risks [12]. Many important questions remain that will help us determine whether social annotation interventions are appropriate in this context: how comments and feedback to be implemented in the consent form are gathered, whether individuals who provide those comments are at risk themselves (for example of exposing too much about themselves), and how (or whether) to "police" information contributed by anonymous others in a form with such a significant impact. Knowing that we may be able to improve certain aspects of the process of deliberating consent by incorporating novel and non-traditional sources of information, however, obligates us as a community to explore social annotation interventions further.

\section{LIMITATIONS AND FUTURE RESEARCH}

While this study demonstrates how exposure to computersupported social annotations impacts individuals' perceptions in the context of informed consent, it has a number of limitations.

Though we believe that the demonstrated increase in the perception of being informed suggests that social annotations can benefit prospective participants, the experiment was structured to study the effects of exposure to annotations on participants' perceptions, and did not examine whether they objectively benefitted from the intervention. Future research is needed to explore whether improvements in the perception of making an informed decision we observed result in quantifiable and objective improvements in the process of analyzing complex consent forms, and whether it results in objectively "better" outcomes for the individual.

Furthermore, participants were led to believe that the comments were provided by former study participants, and behaved without additional information on the accuracy or the bias of the comments. As discussed earlier, individuals may be exposed to, and therefore influenced by, comments that contain wrong information or substantial bias. This is particularly true for the most vulnerable segment of the population - those that are least likely to protect their privacy (as found in this study). Future studies should explore the impact of erroneous information and bias on participants' deliberative processes and consent decisions.

Finally, a number of high-level questions remain for further investigation. In terms of extending research on the effect of exposure to social information on user behavior, there is a need to understand the impact of information on participants' perception and decisions when annotations are not balanced in valence, and to understand if this information leads users to make objectively better decisions for themselves. On the other hand, evaluating the effect of creating and actively engaging with social annotation on user behavior lead requires us to understand how to solicit meaningful content from participants, what motivates individuals to contribute content, what privacy issues are associated with contributing and accessing health-related information, and whether and how to police usercontributed information.

\section{CONCLUSIONS}

Electronic consent has become increasingly popular in Internet research in general and biomedical research in particular. The work presented here explores the effects of adding a computer-supported social dimension to the consent deliberation process. We find that exposure to social annotations results in participants feeling that their decision was more informed, but simultaneously less confident in their understanding of the genomics material presented in the consent form as well as less trusting of the organization soliciting the consent. Based on these findings, we assert that augmenting the consent deliberation process 
with multiple voices enable individuals to capitalize on the knowledge of others, which brings to light questions, problems, and concerns they may not have considered on their own. Thus, social annotations can help people make informed consent decisions that are right for them according to their values.

The study has implications for the design of electronic consent forms. Rather than designing electronic forms that contain simple re-iteration of traditional consent elements, online consent should leverage uniquely available features of digital contexts such as social annotation that can contribute to a more effective deliberation process where users consider various perspectives on what information is important for their consent. The findings also call for future research in $\mathrm{CSCW}$, which may extend the research on collaborative online consent forms to examine the role of novel user generated sources of information, and develop new measures and indicators for evaluating social informed consent.

\section{ACKNOWLEDGMENTS}

This work was partially funded by National Science Foundation grants IIS-1017693 and IIS-1422706.

\section{REFERENCES}

1. 23andMe Consent Form. Retrieved May 1, 2015 from https://www.23andme.com/about/consent/

2. Patricia Agre and Bruce Rapkin. 2003. Improving informed consent: a comparison of four consent tools. IRB: Ethics \& Human Research.

3. Apple ResearchKit. Retrieved May 1, 2015 from http://www.apple.com/researchkit/

4. Christoph Bartneck, Andreas Duenser, Elena Moltchanova, and Karolina Zawieska. 2015. Comparing the Similarity of Responses Received from Studies in Amazon's Mechanical Turk to Studies Conducted Online and with Direct Recruitment. PloS one $10,4$.

5. Eric Baumer, Sherri Katz, Jill Freeman, et al. 2012. Prescriptive persuasion and open-ended social awareness: expanding the design space of mobile health. Proceedings of the ACM conference on Computer Supported Cooperative Work: 475-484.

6. Michael Bernstein, Desney Tan, Greg Smith, Mary Czerwinski, and Eric Horvitz. 2009. Collabio: a game for annotating people within social networks. Proceedings of the ACM symposium on User interface software and technology: 97-100.

7. Tom Buchanan, Carina Paine, Adam Joinson, and UlfDietrich Reips. 2007. Development of measures of online privacy concern and protection for use on the Internet. Journal of the American Society for Information Science and Technology 58, 2: 157-165.

8. Ciro Cattuto, Alain Barrat, Andrea Baldassarri, Gregory Schehr, and Vittorio Loreto. 2009. Collective dynamics of social annotation. Proceedings of the National Academy of Sciences 106, 26: 10511-10515.

9. Robert Cialdini, and Noah Goldstein. 2004. Social influence: Compliance and conformity. Annual Review of Psychology 55: 591-621.

10. Rob Cross, Ronald Rice, and Andrew Parker. 2001. Information seeking in social context. IEEE Transactions on Systems, Man, and Cybernetics, Part C 31, 4: 438-448.

11. Rob Cross and Lee Sproull. 2004. More than an answer: Information relationships for actionable knowledge. Organization Science 15, 4: 446-462.

12. Charles Culver and Bernard Gert. 1982. Philosophy in Medicine: Conceptual and Problems Medicine and Psychiatry. Wadsworth.

13. Sauvik Das, Tiffany Kim, Laura Dabbish, and Jason Hong. 2014. The effect of social influence on security sensitivity. In Proc. SOUPS.

14. Sauvik Das, Adam Kramer, Laura Dabbish, and Jason Hong. 2015. The Role of Social Influence In Security Feature Adoption. Proceedings of the ACM Conference on Computer Supported Cooperative Work. 1416-1426.

15. Graham Dresden and Andrew Levitt. 2001. Modifying a standard industry clinical trial consent form improves patient information retention as part of the informed consent process. Academic Emergency Medicine 8, 3: 246-252.

16. Thomas Duffy, and Paula Kabance. 1982. Testing a readable writing approach to text revision. Journal of Educational Psychology 74, 5: 733.

17. Laura Dunn, Laurie Lindamer, Barton Palmer, et al. 2002. Improving understanding of research consent in middle-aged and elderly patients with psychotic disorders. The American Journal of Geriatric Psychiatry 10, 2: 142-150.

18. Bente Elkjaer. 2003. Social learning theory. The Blackwell handbook of organizational learning and knowledge management. 38-53.

19. Brynn Evans, Sanjay Kairam, and Peter Pirolli. 2010. Do your friends make you smarter?. Information Proc. \& Management 46, 6: 679-692.

20. Rosta Farzan, Laura Dabbish, Robert Kraut, and Tom Postmes. 2011. Increasing commitment to online communities by designing for social presence. Proceedings of the ACM conference on Computer supported cooperative work. 321-330.

21. Food and Drug Administration. 2015. Use of Electronic Informed Consent in Clinical Investigations Questions and Answers Guidance for Industry.

22. Matthew Feinberg, Robb Willer, Jennifer Stellar, and Dacher Keltner. 2012. The virtues of gossip. Journal of personality and social psychology 102, 5: 1015 
23. James Flory and Ezekial Emanuel. 2004. Interventions to improve research participants' understanding in informed consent for research: a systematic review. JAMA 292, 13: 1593-1601.

24. Batya Friedman, Edward Felten, and Lynette Millett. 2000. Informed consent online. CSE Technical Report.

25. Ian Fureman, Kathleen Meyers, Thomas McLellan, David Metzger, and George Woody. 1997. Evaluation of a video-supplement to informed consent. AIDS Education and Prevention 9, 4: 330-341. education and prevention: official publication of the International Society for AIDS Education 9, 4, 330-341.

26. Fei Gao. 2013. A case study of using a social annotation tool to support collaboratively learning. The Internet and Higher Education 17: 76-83.

27. Bradford Gray, Robert Cooke, and Arnold Tannenbaum. 1978. Research involving human subjects. Science 201, 4361: 1094-1101.

28. Nan Hu, Ling Liu, and Jie Zhang. 2008. Do online reviews affect product sales?. Information Technology and Management 9, 3: 201-214.

29. Aniket Kittur, Andrew Peters, Abdigani Diriye, and Michael Bove. 2014. Standing on the schemas of giants: socially augmented information foraging. Proceedings of the ACM conference on Computer supported cooperative work, 999-1010.

30. Shawn Kneipp, Barbara Lutz, and Deirdra Means. 2009. Reasons for enrollment, the informed consent process, and trust among low-income women participating in a community-based participatory research study. Public Health Nursing 26, 4: 362-369.

31. Robert Kraut, Judith Olson, Mahzarin Banaji, Amy Bruckman, Jeffrey Cohen, and Mick Couper. 2004. Psychological research online: report of Board of Scientific Affairs' Advisory Group on the Conduct of Research on the Internet. American psychologist 59, 2: 105.

32. Chinmay Kulkarni and Ed Chi. 2013. All the news that's fit to read: a study of social annotations for news reading. In Proceedings of the SIGCHI Conference on Human Factors in Computing Systems: 2407-2416.

33. Hilary Llewellyn-Thomas, Elaine Thiel, et al. 1995. Presenting clinical trial information. Patient education and counseling 25, 2: 97-107.

34. Todd McElroy and Keith Dowd. 2007. Susceptibility to anchoring effects. Judgment and Decision Making 2, 1: 48-53.

35. Harrison McKnight, Nancy Lankton, and John Tripp. 2011. Social networking information disclosure and continuance intention. IEEE Hawaii International Conference on System Sciences.
36. David Mechanic. 1994. Trust and informed consent to rationing. The Milbank Quarterly: 217-223.

37. David Mechanic. 1998. The functions and limitations of trust in the provision of medical care. Journal of Health Politics, Policy and Law 23, 4: 661-686.

38. Lynette Millett, Batya Friedman, and Edward Felten. 2001. Cookies and web browser design: toward realizing informed consent online. Proceedings of the SIGCHI conference on Human factors in computing systems: 46-52.

39. Gary Morrow. 1980. How readable are subject consent forms? JAMA 244, 1: 56-58.

40. Dean Murphy, Z. O'Keefe, and Alexander Kaufman. 1999. Improving comprehension and recall of information for an HIV vaccine trial among women at risk for HIV. AIDS Education and Prevention 11,5: 389-399.

41. Les Nelson, Christopher Held, Peter Pirolli, Lichan Hong, Diane Schiano, and Ed Chi. 2009. With a little help from my friends: examining the impact of social annotations in sensemaking tasks. In Proceedings of the SIGCHI conference on human factors in computing systems: 1795-1798.

42. Oded Nov and Ofer Arazy. 2015. Asymmetric recommendations: the interacting effects of social ratings' direction and strength on users' ratings. Proceedings of ACM Conference on Recommender Systems.

43. Office for Human Research Protection. Informed Consent Checklist - Basic and Additional Elements. http://www.hhs.gov/ohrp/policy/consentckls.html

44. Gabriele Paolacci, Jesse Chandler, and Panagiotis Ipeirotis. 2010. Running experiments on amazon mechanical turk. Judgment and Decision Making 5, 5: 411-419.

45. Daniel Power. 2008. Decision support systems: a historical overview. In Handbook on Decision Support Systems 1: 121-140.

46. Project, Personal Genome Education Retrieved May 1, 2015 from http://www.pged.org

47. Orit Shaer, Oded Nov, Johanna Okerlund, Martina Balestra, Elizabeth Stowell, Laura Ascher, Joanna Bi Claire Schlenker, and Madeleine Ball (2015). Informing the Design of Direct-to-Consumer Interactive Personal Genomics Reports. Journal of medical Internet research, 17(6).

48. Shaer, O., \& Nov, O. (2014). HCI for personal genomics. ACM Interactions, 21(5), 32-37.

49. Baba Shiv, Julie Britton, and John Payne. 2004. Does elaboration increase or decrease the effectiveness of negatively versus positively framed messages? Journal of Consumer Research 31, 1: 199-208. 
50. Nancy Staggers and Anthony Norcio. 1993. Mental models: concepts for human-computer interaction research. International Journal of Man-machine studies 38, 4: 587-605.

51. Paul Stiles, Norman Poythress, Alicia Hall, Diana Falkenbach, and Robyn Williams. 2001. Improving understanding of research consent disclosures among persons with mental illness. Psychiatric Services 52, 6: 780-785.

52. Amos Tversky and Daniel Kahneman. 1974. Judgment under uncertainty: Heuristics and biases. Science 185, 4157: 1124-1131.

53. Peter Ubel and George Loewenstein. 1997. The role of decision analysis in informed consent. Social science \& medicine 44, 5: 647-656.

54. Alan Westin, Danielle Maurici, Price Waterhouse, and Louis Harris. 1998. E-commerce \& privacy. Privacy \& American Business.

55. Qiang Ye, Rob Law, Bin Gu, and Wei Chen. 2011. The influence of user-generated content on traveler behavior. Computers in Human Behavior 27: 634-639. 